\title{
Well-posedness of the Cauchy problem for nonlinear parabolic equations with variable density in the hyperbolic space
}

\author{
Fabio Punzo
}

\begin{abstract}
We investigate the well-posedness of the Cauchy problem for a class of nonlinear parabolic equations with variable density in the hyperbolic space. We state sufficient conditions for uniqueness or nonuniqueness of bounded solutions, depending on the behavior of the density at infinity. Nonuniqueness relies on the prescription at infinity of suitable conditions of Dirichlet type, and possibly inhomogeneous.
\end{abstract}

Mathematics Subject Classification (2000). 35A02, 35K59, 35K65, 35K67, 35J35.

Keywords. Singular nonlinear parabolic equations, Laplace-Beltrami operator, Well-posedness, Comparison principles, Sub-supersolutions.

\section{Introduction}

We study uniqueness and nonuniqueness of bounded solutions to nonlinear Cauchy problems with variable density of the following form:

$$
\begin{cases}a \partial_{t} u=\Delta_{g}[\phi(u)] & \text { in } \mathbb{H}^{n} \times(0, T]=: H_{T} \\ u=u_{0} & \text { in } \mathbb{H}^{n} \times\{0\}\end{cases}
$$

here $\mathbb{H}^{n}$ is the $n$-dimensional hyperbolic space $(n \geq 2), \Delta_{g}$ is the LaplaceBeltrami operator in $\mathbb{H}^{n}$, a, referred to as a density, is a positive function of the space variables, $u_{0}$ is bounded. A typical choice for the function $\phi$ is $\phi(u)=|u|^{m-1} u$ with $m \geq 1$. More precisely, we always assume the following:

$$
\begin{cases}\text { (i) } & a \in C\left(\mathbb{H}^{n}\right), a>0 \text { in } \mathbb{H}^{n} ; \\ \text { (ii) } & \phi \in C^{1}(\mathbb{R}), \phi(0)=0, \phi^{\prime}(s)>0 \text { for any } s \in \mathbb{R} \backslash\{0\}, \\ & \phi^{\prime} \text { decreasing in }(-\delta, 0) \text { and increasing in }(0, \delta), \\ & \text { if } \phi^{\prime}(0)=0(\delta>0) ; \\ \text { (iii) } u_{0} \in C\left(\mathbb{H}^{n}\right), u_{0} \text { is bounded. }\end{cases}
$$


The Cauchy problem (1.1) can be regarded as the counterpart in $\mathbb{H}^{n}$ of the Cauchy problem

$$
\begin{cases}a \partial_{t} u=\Delta[\phi(u)] & \text { in } \mathbb{R}^{n} \times(0, T] \\ u=u_{0} & \text { in } \mathbb{R}^{n} \times\{0\},\end{cases}
$$

which has been the object of detailed investigations (e.g., see $[2,4,6,13,18,21])$. It is worth mentioning that problem (1.2) arises in situations of physical interest (see [15]) in a wide variety of fields that involve diffusion processes. When such diffusion processes are considered on the hyperbolic space $\mathbb{H}^{n}$, then it is natural to introduce problem (1.1) to describe them.

In the literature, the case of linear diffusion has been particularly addressed. In fact, problem (1.1) with $a \equiv 1$ and $\phi(u)=u$ has been largely studied (e.g., see [5,7-10] and references therein) by means of analytical and probabilistic methods.

Notice that also qualitative properties of solutions to semilinear elliptic equations on $\mathbb{H}^{n}$ have been investigated (see $[3,16]$ ). Of course, parabolic and elliptic equations on the hyperbolic space have attracted so much attention, since $\mathbb{H}^{n}$ is the model of complete Riemannian manifolds with constant negative sectional curvature (see $[1,8]$ ).

Recall that, as is well-known, problem (1.2) is well-posed in the class of bounded solutions when $a$ is bounded and $n \leq 2$ (see [11]), or when $n \geq 3$ and $a$ is constant (see $[2,4,12]$ ). Moreover, for $n \geq 3$, uniqueness of bounded solutions to problem (1.2), not satisfying any extra constraints at infinity, has been showed if $a(x) \rightarrow 0$ slowly, or $a(x)$ is bounded from below by a positive constant or it goes to infinity as $|x| \rightarrow \infty$ (see $[13,19])$.

On the contrary, for $n \geq 3$, existence of bounded solutions to problem (1.2), satisfying at infinity some additional conditions, has been proved, if $a(x) \rightarrow 0$ sufficiently fast as $|x| \rightarrow \infty$ (see $[6,13,14,19,21])$. Clearly, these existence results imply nonuniqueness of bounded solutions to the Cauchy problem (1.2).

Such uniqueness and nonuniqueness results have also a deep probabilistic interpretation in the case $\phi(u)=u$. In fact, uniqueness for problem (1.2) holds true, when the Markov process associated to the operator $\frac{1}{a} \Delta$ starting from any point of $\mathbb{R}^{n}$ is recurrent (see [8]), thus, loosely speaking, it cannot attain the infinity, therefore we cannot prescribe conditions at infinity. Instead, when this Markov process is transient, and so it can reach the infinity, we can prescribe some conditions at infinity in a proper sense, whence nonuniqueness follows.

The aim of this paper is to prove the following:

(i) uniqueness of bounded solutions to problem (1.1) not satisfying at infinity any additional constraints, when $a(x)$ is bounded from below by a positive constant or it goes to infinity as $x$ tends to infinity and $n \geq 2$;

(ii) existence of bounded solutions to problem (1.1) satisfying at infinity some extra conditions, when $a(x)$ goes to zero as $x$ tends to infinity and $n \geq 2$. 
Such results extend to the nonlinear problem (1.1) those already established, in [17], for problem (1.1) with $\phi(u)=u$ and $n \geq 2$, by means of different methods which cannot be applied to the nonlinear problem (1.1) with general $\phi$. Moreover, for $a(x) \equiv 1, \phi(u)=u$ and $n \geq 2$, uniqueness of bounded solutions to the Cauchy problem (1.1) has already been obtained in [8]. Finally, let us mention that support properties of solutions to problem (1.1) has been studied in [20], provided that supp $u_{0}$ is compact.

The paper is organized as follows. In Sect. 2 we state the uniqueness and nonuniqueness results, while in Sect. 3 we introduce some preliminary notions of hyperbolic geometry which will be used in the sequel. Finally, in Sects. 4 and 5 we shall prove uniqueness and nonuniqueness results, respectively.

\section{Results}

To state our results, we need to introduce some notations. For any $r>0$ let $B_{r}:=\left\{x \in \mathbb{R}^{n}|| x \mid<r\right\}$; here and hereafter $|\cdot|$ denotes the standard euclidian norm.

In the sequel we shall consider the Poincaré ball model of the hyperbolic space $\mathbb{H}^{n}$. To be specific, we set $\mathbb{H}^{n} \equiv B_{1}$ endowed with the Riemannian metric

$$
g_{i j}:=\frac{4}{\left(1-|x|^{2}\right)^{2}} \delta_{i j} \quad\left(x \in B_{1} ; i, j=1, \ldots, n\right) ;
$$

moreover, let

$$
g^{i j}:=\left(g_{i j}\right)^{-1}, \quad g:=\operatorname{det}\left(g_{i j}\right) \quad(i, j=1, \ldots, n) .
$$

As usual (see [1]), we set

$$
\partial B_{1} \equiv \partial_{\infty} \mathbb{H}^{n} \equiv\{\infty\}
$$

\subsection{Uniqueness results}

At first, let us state a standard existence result of solutions to problem (1.1).

Theorem 2.1. Let assumption $\left(H_{0}\right)$ be satisfied. Then there exists a bounded solution of problem (1.1).

The following uniqueness result will be proved.

Theorem 2.2. Let assumption $\left(H_{0}\right)$ be satisfied. Moreover, suppose that

$$
\left\{\begin{array}{l}
\text { there exist } C_{1}>0 \text { and } \alpha \geq 0 \text { such that } \\
a(x) \geq C_{1}(1-|x|)^{-\alpha} \text { for any } x \in \mathbb{H}^{n} .
\end{array}\right.
$$

Then there exists at most one bounded solution of problem (1.1).

Remark 2.3. Hypothesis $\left(H_{1}\right)$ can be expressed also as follows:

$$
\left\{\begin{array}{l}
\text { there exist } C_{1}>0 \text { and } \alpha \geq 0 \text { such that } \\
a(x) \geq C_{1} e^{\alpha \rho(x)} \quad \text { for any } x \in \mathbb{H}^{n} ;
\end{array}\right.
$$

here $\rho(x)$ denotes the geodesic distance in $\mathbb{H}^{n}$ of $x \in \mathbb{H}^{n}$ from 0 (see (3.1) below). 
Remark 2.4. In Theorem 2.2 hypothesis $\left(H_{1}\right)$ can be replaced by the following weaker condition

$$
\left(H_{1}\right)^{*} \quad\left\{\begin{array}{l}
\text { there exist } \hat{\varepsilon} \in(0,1) \text { and } \underline{a} \in C([0, \hat{\varepsilon}] ;[0,+\infty)) \text { such that } \\
\text { (i) } a(x) \geq \underline{a}(1-|x|)>0 \text { for any } x \in B_{1-\hat{\varepsilon}}, \text { and } \\
\text { (ii) } \int_{0}^{\hat{\varepsilon}} \frac{\underline{a}(\eta)}{\eta} d \eta=\infty .
\end{array}\right.
$$

\subsection{Nonuniqueness results}

We shall prove the following result which regards existence of solutions to problem (1.1) satisfying additional conditions at infinity (see (2.4)).

Theorem 2.5. Let assumption $\left(H_{0}\right)$ be satisfied and $A \in \operatorname{Lip}([0, T])$ with $A(0)=0$. Moreover, suppose that

$$
\left(H_{2}\right) \quad\left\{\begin{array}{l}
\text { there exist } C_{2}>0 \text { and } \alpha<0 \text { such that } \\
a(x) \leq C_{2}(1-|x|)^{-\alpha} \text { for any } x \in \mathbb{H}^{n} .
\end{array}\right.
$$

Then there exists a bounded solution $u$ of problem (1.1) such that

$$
\lim _{|x| \rightarrow 1}|U(x, t)-A(t)|=0
$$

uniformly with respect to $t \in[0, T]$; here

$$
U(x, t):=\int_{0}^{t} \phi(u(x, \tau)) d \tau \quad\left((x, t) \in H_{T}\right) .
$$

Remark 2.6. Hypothesis $\left(H_{2}\right)$ can be expressed also as follows:

$$
\left\{\begin{array}{l}
\text { there exist } C_{2}>0 \text { and } \alpha<0 \text { such that } \\
a(x) \leq C_{2} e^{\alpha \rho(x)} \text { for any } x \in \mathbb{H}^{n}
\end{array}\right.
$$

here $\rho(x)$ denotes the geodesic distance in $\mathbb{H}^{n}$ of $x \in \mathbb{H}^{n}$ from 0 (see (3.1) below).

Consider the following condition:

$$
\left(H_{2}^{*}\right) \quad \text { there exists } x_{0} \in \mathbb{H}^{n} \text { such that } \int_{\mathbb{H}^{n}} G_{g}\left(x_{0}, y\right) a(y) d \mu(y)<\infty,
$$

where $G_{g}$ is the Green function for $\Delta_{g}$ in $\mathbb{H}^{n}$ (see [8]) and $d \mu$ is the Riemannian volume element (see (3.6) below).

Remark 2.7. If assumption $\left(H_{2}\right)^{*}$ is satisfied, then we have $\int_{\mathbb{H}^{n}} G_{g}(x, y) a(y) d \mu$ $(y)<\infty$ for any $x \in \mathbb{H}^{n}$ (see [7]). Furthermore, since

$$
G_{g}(0, x)=\int_{\rho(x)}^{\infty} \frac{d \xi}{(\sinh \xi)^{n-1}} \quad\left(x \in \mathbb{H}^{n} \backslash\{0\}\right)
$$

it is direct to see that hypothesis $\left(H_{2}\right)$ implies $\left(H_{2}\right)^{*}$.

If in Theorem 2.5 assumption $\left(H_{2}\right)$ is replaced by the weaker condition $\left(H_{2}\right)^{*}$, then we obtain next 
Theorem 2.8. Let assumptions $\left(H_{0}\right),\left(H_{2}\right)^{*}$ be satisfied and $A \in \operatorname{Lip}([0, T])$ with $A(0)=0$. Then there exists a sequence $\left\{x_{m}\right\} \subseteq \mathbb{H}^{n},\left|x_{m}\right| \rightarrow 1$ as $m \rightarrow \infty$ and a bounded solution $u$ of problem (1.1) satisfying

$$
\lim _{m \rightarrow+\infty}\left|U\left(x_{m}, t\right)-A(t)\right|=0
$$

uniformly with respect to $t \in[0, T]$, with $U$ defined in (2.6).

Remark 2.9. Clearly, both Theorems 2.5 and 2.8 imply nonuniqueness of bounded solutions to problem (1.1).

\section{Mathematical background}

The geodesic distance between any $x \in \mathbb{H}^{n}$ and 0 is given by

$$
\rho(x):=\int_{0}^{|x|} \frac{2}{1-s^{2}} d s=\log \left(\frac{1+|x|}{1-|x|}\right) \quad\left(x \in \mathbb{H}^{n} \equiv B_{1}\right) ;
$$

therefore

$$
|x|=\tanh \left(\frac{\rho(x)}{2}\right), \quad \frac{2}{1-|x|^{2}}=2\left[\cosh \left(\frac{\rho(x)}{2}\right)\right]^{2} \quad\left(x \in \mathbb{H}^{n}\right) .
$$

For any $r>0$ define

$$
\mathfrak{B}_{r}:=\left\{x \in \mathbb{H}^{n} \mid \rho(x)<r\right\}, \mathfrak{S}_{r}:=\left\{x \in \mathbb{H}^{n} \mid \rho(x)=r\right\} .
$$

Hence for any $r \in(0,1)$

$$
B_{r}=\mathfrak{B}_{\log \left(\frac{1+r}{1-r}\right)}
$$

Let $(\rho, \theta)$ be polar geodesic coordinates in $\mathbb{H}^{n}$. Then we have

$$
d s^{2}:=\sum_{i, j=1}^{n} g_{i j} d x_{i} d x_{j}=d \rho^{2}+(\sinh \rho)^{2} d \theta
$$

moreover,

$$
\Delta_{g}=\frac{\partial^{2}}{\partial \rho^{2}}+(n-1) \operatorname{coth} \rho \frac{\partial}{\partial \rho}+\frac{1}{(\sinh \rho)^{2}} \Delta_{\theta},
$$

where $\Delta_{\theta}$ is the Laplace-Beltrami operator on the $(n-1)$-dimensional sphere of $\mathbb{R}^{n}$.

In $\mathbb{H}^{n}$ the Riemannian volume element is defined by

$$
d \mu:=\sqrt{g} d x_{1} d x_{2} \ldots d x_{n}=\frac{2^{n}}{\left(1-|x|^{2}\right)^{n}} d x_{1} \ldots d x_{n},
$$

$d x_{1} d x_{2} \ldots d x_{n} \equiv d x$ being the Lebesgue measure in $\mathbb{R}^{n}$. Furthermore, let $d \mu^{\prime}$ be the Riemannian area element on submanifold of $\mathbb{H}^{n}$ of codimension 1 . We have for any $r>0$

$$
\mu^{\prime}\left(\mathfrak{S}_{r}\right)=\omega_{n}(\sinh r)^{n-1},
$$

where $\omega_{n}$ is the area of the unit sphere of $\mathbb{R}^{n}$. 
For every $x \in \mathbb{H}^{n}$, let $T_{x} \mathbb{H}^{n}$ denote the tangent space to $\mathbb{H}^{n}$ at $x$. As usual, let $\left(\frac{\partial}{\partial x_{1}}, \ldots, \frac{\partial}{\partial x_{n}}\right)$ be the standard basis of $T_{x} \mathbb{H}^{n}$. The inner Riemannian product $\langle\cdot, \cdot\rangle_{g}$ for every $x \in \mathbb{H}^{n}$ is given by:

$$
\langle\beta, \xi\rangle_{g} \equiv\langle\beta, \xi\rangle_{g, x}=\sum_{i, j=1}^{n} g_{i j} \beta_{i} \xi_{j}
$$

for any $\beta, \xi \in T_{x} \mathbb{H}^{n}$, with $\beta=\sum_{i=1}^{n} \beta_{i} \frac{\partial}{\partial x_{i}}$ and $\xi=\sum_{i=1}^{n} \xi_{i} \frac{\partial}{\partial x_{i}}$ for some $\left(\beta_{1}, \ldots, \beta_{n}\right) \in \mathbb{R}^{n}$ and $\left(\xi_{1}, \ldots, \xi_{n}\right) \in \mathbb{R}^{n}$.

Recall that the gradient $\nabla_{g} u \equiv\left(\left(\nabla_{g} u\right)_{1}, \ldots,\left(\nabla_{g} u\right)_{n}\right)$ is given by

$$
\left(\nabla_{g} u\right)_{i}:=\sum_{j=1}^{n} g^{i j} \frac{\partial u}{\partial x_{j}} \quad(i=1, \ldots, n)
$$

furthermore,

$$
\|\nu\|_{g}:=\sqrt{\langle\nu, \nu\rangle_{g}} \quad\left(\nu \in T_{x} \mathbb{H}^{n}, x \in \mathbb{H}^{n}\right) .
$$

Let us make the following definition.

Definition 3.1. A solution of problem (1.1) is a function $u \in C\left(\mathbb{H}^{n} \times(0, T]\right)$ such that

$$
\begin{aligned}
\int_{0}^{\tau} \int_{\Omega_{1}}\left\{a u \partial_{t} \psi+\phi(u) \Delta_{g} \psi\right\} d \mu d t= & \int_{\Omega_{1}} a\left[u(x, \tau) \psi(x, \tau)-u_{0}(x) \psi(x, 0)\right] d \mu \\
& +\int_{0}^{\tau} \int_{\partial \Omega_{1}} \phi(u)\left\langle\nabla_{g} \psi, \nu\right\rangle_{g} d \mu^{\prime} d t
\end{aligned}
$$

for any precompact set $\Omega_{1} \subseteq \mathbb{H}^{n}$ with smooth boundary $\partial \Omega_{1}, \bar{\Omega}_{1} \subseteq \mathbb{H}^{n}, \tau \in$ $(0, T], \psi \in C^{2,1}\left(\overline{\Omega_{1}} \times[0, \tau]\right), \psi \geq 0, \psi=0$ in $\partial \Omega_{1} \times[0, \tau] ; \nu$ denotes the outer normal to $\Omega_{1}$ satisfying $\|\nu\|_{g}=1$.

Supersolutions (subsolutions) of (1.1) are defined replacing "=" by " $\leq$ " (" $\geq$ ", respectively) in (3.10).

\section{Proof of Theorems 2.1 and 2.2}

Proof of Theorem 2.1. The conclusion follows by the same argument as in the proof of Theorem 2.5 in [18].

In what follows we regard $B_{1-\varepsilon}(\varepsilon \in(0,1))$ as a subset of $\mathbb{H}^{n}$, in view of the identification $\mathbb{H}^{n} \equiv B_{1}$. For any $\varepsilon \in(0,1)$ the operator $\Delta_{g}$ is uniformly elliptic in $B_{1-\varepsilon}$, hence there exists a unique classical solution $\psi_{\varepsilon}$ of the elliptic problem:

$$
\begin{cases}\Delta_{g} U=-F & \text { in } B_{1-\varepsilon} \\ U=0 & \text { on } \partial B_{1-\varepsilon},\end{cases}
$$

where $F \in C_{0}^{\infty}\left(B_{1}\right)$. In the proof of Theorem 2.2 we will use next lemma. 
Lemma 4.1. Let assumption $\left(H_{0}\right)$ be satisfied, $\varepsilon_{0} \in(0,1)$. For any $\varepsilon \in\left(0, \varepsilon_{0}\right)$ let $\psi_{\varepsilon}$ be the solution to problem (4.1) with $F \in C^{\infty}\left(B_{1}\right), F \geq 0$, supp $F \subseteq B_{1-\varepsilon_{0}}$. Then the following statements hold true:

(i) for any $\varepsilon_{1}, \varepsilon_{2} \in\left(0, \varepsilon_{0}\right), \varepsilon_{1} \geq \varepsilon_{2}$ we have

$$
0<\psi_{\varepsilon_{1}} \leq \psi_{\varepsilon_{2}} \text { in } B_{1-\varepsilon_{1}}
$$

(ii) there exists $C>0$ such that for any $\varepsilon \in\left(0, \varepsilon_{0} / 2\right)$ we have

$$
-C\left(\frac{1-|x|^{2}}{2|x|}\right)^{n-1} \leq\left\langle\nabla_{g} \psi_{\varepsilon}, \nu_{\varepsilon}\right\rangle_{g}<0 \text { for any } x \in \partial B_{1-\varepsilon}
$$

$\nu_{\varepsilon}$ denoting the outer normal to $B_{1-\varepsilon}$ at $\partial B_{1-\varepsilon}$, with $\left\|\nu_{\varepsilon}\right\|_{g}=1$;

(iii) there exist $\bar{C}>0$ and $\bar{\varepsilon} \in\left(0, \varepsilon_{0}\right)$ such that

$$
\psi_{0}(x) \geq \bar{C}\left(1-|x|^{2}\right)^{n-1} \quad \text { for any } x \in B_{1} \backslash B_{1-\bar{\varepsilon}},
$$

where

$$
\psi_{0}:=\lim _{\varepsilon \rightarrow 0} \psi_{\varepsilon} \quad \text { in } B_{1} .
$$

Proof. (i) By the strong maximum principle $\psi_{\varepsilon}>0$ in $B_{1-\varepsilon}$ for any $\varepsilon \in$ $\left(0, \varepsilon_{0}\right)$; hence the function $\psi_{\varepsilon_{1}}-\psi_{\varepsilon_{2}}\left(0<\varepsilon_{2} \leq \varepsilon_{1}<\varepsilon_{0}\right)$ is a subsolution of problem

$$
\begin{cases}\Delta_{g} U=0 & \text { in } B_{1-\varepsilon_{1}} \\ U=0 & \text { on } \partial B_{\varepsilon_{1}} .\end{cases}
$$

Then again by the maximum principle we get (4.2).

(ii) Clearly, by the strong maximum principle there holds $\left\langle\nabla \psi_{\varepsilon}, \nu_{\varepsilon}\right\rangle_{\mathbb{R}^{n}}<0$ on $\partial B_{\varepsilon}$ for any $\varepsilon \in\left(0, \varepsilon_{0} / 2\right)$. Hence

$$
\left\langle\nabla_{g} \psi, \nu_{\varepsilon}\right\rangle_{g}<0
$$

It is well-known that (see [8])

$$
\psi_{0}(x)=\int_{\mathbb{H}^{n}} G_{g}(x, y) F(y) d \mu(y) \quad\left(x \in \mathbb{H}^{n} \equiv B_{1}\right),
$$

where $\psi_{0}$ is defined in (4.5). From $(i)$, for any $\varepsilon \in\left(0, \varepsilon_{0}\right)$, we have

$$
\psi_{\varepsilon} \leq \psi_{0} \quad \text { in } B_{1-\varepsilon} .
$$

For any $\varepsilon \in\left(0, \varepsilon_{0}\right)$ let us introduce the problem

$$
\begin{cases}\Delta_{g} U=0 & \text { in } B_{1-\varepsilon} \backslash B_{1-\varepsilon_{0}} \\ U=0 & \text { on } \partial B_{1-\varepsilon} \\ U=M & \text { on } \partial B_{1-\varepsilon_{0}}\end{cases}
$$

where $M:=\max _{\partial B_{1-\varepsilon_{0}}} \psi_{0}$.

Observe that (see [8], Sect. 4.2) the function

$$
\check{Z}(x):=\int_{\rho(x)}^{+\infty} \frac{1}{(\sinh \xi)^{n-1}} d \xi \quad\left(x \in \mathbb{H}^{n}\right)
$$


solves

$$
\Delta_{g} \check{Z}=0 \quad \text { in } \mathbb{H}^{n} \backslash\{0\}
$$

Define

$$
\begin{gathered}
Z(x) \equiv Z(\rho(x)):=C_{\varepsilon}\left[\int_{\rho(x)}^{+\infty} \frac{1}{(\sinh \xi)^{n-1}} d \xi-\int_{\tilde{\rho}(1-\varepsilon)}^{+\infty} \frac{1}{(\sinh \xi)^{n-1}} d \xi\right] \\
\left(x \in B_{1-\varepsilon} \backslash B_{1-\varepsilon_{0}}\right),
\end{gathered}
$$

where

$$
\begin{gathered}
C_{\varepsilon}:=\frac{M}{\int_{\tilde{\rho}\left(1-\varepsilon_{0}\right)}^{+\infty} \frac{1}{(\sinh \xi)^{n-1}} d \xi-\int_{\tilde{\rho}(1-\varepsilon)}^{+\infty} \frac{1}{(\sinh \xi)^{n-1}} d \xi}, \\
\tilde{\rho}(s):=\log \left(\frac{1+s}{1-s}\right) \quad(s \in[0,1)) .
\end{gathered}
$$

From (4.10) immediately follows that $Z$ is a supersolution to problem (4.9) for any $\varepsilon \in\left(0, \varepsilon_{0} / 2\right)$. On the other hand, $\psi_{\varepsilon}\left(\varepsilon \in\left(0, \varepsilon_{0}\right)\right)$ is a subsolution to the same problem. By comparison principles we get for any $\varepsilon \in\left(0, \varepsilon_{0} / 2\right)$

$$
Z \geq \psi_{\varepsilon} \quad \text { in } ; B_{1-\varepsilon} \backslash B_{1-\varepsilon_{0}} .
$$

Since for any $\varepsilon \in\left(0, \varepsilon_{0} / 2\right)$

$$
Z=\psi_{\varepsilon}=0 \quad \text { on } \partial B_{1-\varepsilon}
$$

we can infer that

$$
\begin{aligned}
\left\langle\nabla_{g} \psi_{\varepsilon}, \nu_{\varepsilon}\right\rangle_{g} & \geq\left\langle\nabla_{g} Z, \nu_{\varepsilon}\right\rangle_{g}=\frac{\partial Z(\rho)}{\partial \rho}=-\frac{C_{\varepsilon}}{[\sinh (\rho(x))]^{n-1}} \\
& \geq-C_{\varepsilon_{0} / 2}\left(\frac{1-|x|^{2}}{2|x|}\right)^{n-1} \quad\left(x \in \partial B_{1-\varepsilon}\right),
\end{aligned}
$$

This combined with (4.7) gives (4.3).

(iii) Let $0<\varepsilon_{1}<\varepsilon_{2}<\varepsilon_{0}$. Clearly, the function $\psi_{\varepsilon_{1}}$ is a supersolution to problem

$$
\begin{cases}\Delta_{g} U=-F & \text { in } B_{1-\varepsilon_{1} \backslash B_{1-\varepsilon_{2}}} \\ U=0 & \text { on } \partial B_{1-\varepsilon_{1}} \\ U=m & \text { on } \partial B_{1-\varepsilon_{2}},\end{cases}
$$

where $m:=\min _{\partial B_{1-\varepsilon_{2}}} \psi_{\varepsilon_{1}}$. From $(i)$ it follows that for any $0<\varepsilon<\varepsilon_{1}$

$$
\psi_{\varepsilon} \geq \psi_{\varepsilon_{1}} \quad \text { in } \quad B_{1-\varepsilon_{1}} \backslash B_{1-\varepsilon_{2}} .
$$


Then, for any $\varepsilon \in\left(0, \varepsilon_{2}\right), \psi_{\varepsilon}$ is a supersolution to problem

$$
\begin{cases}\Delta_{g} U=-F & \text { in } B_{1-\varepsilon} \backslash B_{1-\varepsilon_{2}} \\ U=0 & \text { on } \partial B_{1-\varepsilon} \\ U=m & \text { on } \partial B_{1-\varepsilon_{2}} .\end{cases}
$$

Observe that

$$
\tilde{\psi}_{\varepsilon}:=m \frac{\int_{\rho(x)}^{+\infty} \frac{1}{(\sinh \xi)^{n-1}} d \xi-\int_{\tilde{\rho}(1-\varepsilon)}^{+\infty} \frac{1}{(\sinh \xi)^{n-1}} d \xi}{\int_{\tilde{\rho}\left(1-\varepsilon_{2}\right)}^{+\infty} \frac{1}{(\sinh \xi)^{n-1}} d \xi-\int_{\tilde{\rho}(1-\varepsilon)}^{+\infty} \frac{1}{(\sinh \xi)^{n-1}} d \xi} \quad\left(x \in B_{1-\varepsilon} \backslash B_{1-\varepsilon_{2}}\right)
$$

is a subsolution to problem (4.12). By comparison principles we have

$$
\tilde{\psi}_{\varepsilon} \leq \psi_{\varepsilon} \quad \text { in } B_{1-\varepsilon} \backslash B_{1-\varepsilon_{2}} .
$$

Sending $\varepsilon \rightarrow 0$ we obtain

$$
\frac{m}{\int_{\tilde{\rho}\left(1-\varepsilon_{2}\right)}^{+\infty} \frac{1}{(\sinh \xi)^{n-1}} d \xi} \int_{\rho(x)}^{+\infty} \frac{1}{(\sinh \xi)^{n-1}} d \xi \leq \psi_{0} \quad \text { in } B_{1} \backslash B_{1-\varepsilon_{2}} .
$$

Then easy computations yield (4.4) with $\bar{\varepsilon}=\varepsilon_{2}$.

Let us prove the following

Lemma 4.2. Let assumptions $\left(H_{0}\right),\left(H_{1}\right)^{*}$ be satisfied. Let $v_{1}, v_{2}$ be any two bounded solutions of problem (1.1) such that $v_{1} \geq v_{2}$ in $H_{T}$. Then

$$
\liminf _{\varepsilon \rightarrow 0} \varepsilon^{n-1} \int_{0}^{T} \int_{\partial B_{1-\varepsilon}}\left\{\phi\left(v_{1}\right)-\phi\left(v_{2}\right)\right\} d \mu^{\prime} d t=0 .
$$

Proof. From problem (1.1) we have

$$
\begin{cases}a\left(\partial_{t} v_{1}-\partial_{t} v_{2}\right)=\Delta\left[\phi\left(v_{1}\right)-\phi\left(v_{2}\right)\right] & \text { in } H_{T} \\ v_{1}-v_{2}=0 & \text { in } \mathbb{H}^{n} \times\{0\} .\end{cases}
$$

Then equality (3.10) with $\Omega_{1}=B_{1-\varepsilon}\left(\varepsilon \in\left(0, \varepsilon_{0} / 2\right)\right), \tau \in(0, T]$ yields

$$
\begin{array}{r}
\int_{B_{1-\varepsilon}} a(x)\left[v_{1}(x, \tau)-v_{2}(x, \tau)\right] \psi_{\varepsilon}(x) d \mu+\int_{0}^{\tau} \int_{B_{1-\varepsilon}}\left[\phi\left(v_{1}\right)-\phi\left(v_{2}\right)\right] F(x) d \mu d t \\
=-\int_{0}^{\tau} \int_{\partial B_{1-\varepsilon}}\left\{\phi\left(v_{1}\right)-\phi\left(v_{2}\right)\right\}\left\langle\nabla_{g} \psi_{\varepsilon}, \nu_{\varepsilon}\right\rangle_{g} d \mu^{\prime} d t
\end{array}
$$

where $\psi_{\varepsilon}$ denotes the solution of problem (4.1). Set

$$
\varphi(\varepsilon):=\int_{0}^{T} \int_{\partial B_{1-\varepsilon}}\left\{\phi\left(v_{1}\right)-\phi\left(v_{2}\right)\right\} d \mu^{\prime} d t \quad\left(\varepsilon \in\left(0, \varepsilon_{0} / 2\right)\right) .
$$

Suppose, by absurd, that

$$
\liminf _{\varepsilon \rightarrow 0} \varepsilon^{n-1} \varphi(\varepsilon) \geq \gamma
$$


for some $\gamma>0$; then there exists $\check{\varepsilon} \in\left(0, \varepsilon_{0} / 2\right)$ such that

$$
\varepsilon^{n-1} \varphi(\varepsilon) \geq \frac{\gamma}{2} \quad \text { for any } \varepsilon \in(0, \check{\varepsilon}) .
$$

Let $M:=\max \left\{\sup _{H_{T}}\left|v_{1}\right|, \sup _{H_{T}}\left|v_{2}\right|\right\}$. Since $F \geq 0, v_{1} \geq v_{2}$ and $\left(H_{0}\right)-(i i)$ holds true, from (4.3) and (4.15) we have

$$
\begin{aligned}
& \int_{0}^{T} \int_{B_{1-\varepsilon}} a(x)\left[v_{1}(x, \tau)-v_{2}(x, \tau)\right] \psi_{\varepsilon}(x) d \mu d \tau \\
& \leq \int_{0}^{T} \int_{0}^{\tau} \int_{\partial B_{1-\varepsilon}}\left\{\phi\left(v_{1}\right)-\phi\left(v_{2}\right)\right\}\left|\left\langle\nabla_{g} \psi_{\varepsilon}, \nu_{\varepsilon}\right\rangle_{g}\right| d \mu^{\prime} d t d \tau \\
& \leq 2 \max _{-M \leq r \leq M}|\phi(r)| \int_{0}^{T} \int_{0}^{\tau} \int_{\partial B_{1-\varepsilon}}\left|\left\langle\nabla_{g} \psi_{\varepsilon}, \nu_{\varepsilon}\right\rangle_{g}\right| d \mu^{\prime} d t d \tau \\
& \leq 2 \max _{-M \leq r \leq M}|\phi(r)| T^{2} \mu^{\prime}\left(\partial B_{1-\varepsilon}\right) C\left[\frac{(2-\varepsilon) \varepsilon}{2(1-\varepsilon)}\right]^{n-1} \\
& =2 \max _{-M \leq r \leq M}|\phi(r)| T^{2} C \mu^{\prime}\left(\mathfrak{S}_{\log \left(\frac{2-\varepsilon}{\varepsilon}\right)}\right)\left[\frac{(2-\varepsilon) \varepsilon}{2(1-\varepsilon)}\right]^{n-1} \\
& =2 \max _{-M \leq r \leq M}|\phi(r)| T^{2} C \omega_{n}\left[\frac{2(1-\varepsilon)}{(2-\varepsilon) \varepsilon}\right]^{n-1}\left[\frac{(2-\varepsilon) \varepsilon}{2(1-\varepsilon)}\right]^{n-1} \\
& =2 \max _{-M \leq r \leq M}|\phi(r)| T^{2} C \omega_{n}
\end{aligned}
$$

for any $\varepsilon \in\left(0, \varepsilon_{0} / 2\right)$; here use of (3.7) has been made.

Letting $\varepsilon \rightarrow 0$ in (4.17), by (4.2), (4.5) and the monotone convergence theorem we have

$$
\int_{0}^{T} \int_{\Omega} a(x)\left[v_{1}(x, \tau)-v_{2}(x, \tau)\right] \psi_{0}(x) d x d \tau \leq 2 \max _{-M \leq r \leq M}|\phi(r)| T^{2} C \omega_{n} .
$$

On the other hand, we have

$$
\begin{aligned}
& \int_{0}^{T} \int_{B_{1}} a(x)\left[v_{1}(x, \tau)-v_{2}(x, \tau)\right] \psi_{0}(x) d \mu d \tau \\
& \geq \frac{1}{L} \int_{0}^{T} \int_{B_{1} \backslash B_{1-\varepsilon}} a(x)\left[\phi\left(v_{1}\right)-\phi\left(v_{2}\right)\right] \psi_{0}(x) d \mu d \tau ;
\end{aligned}
$$

here $\tilde{\varepsilon}:=\min \{\hat{\varepsilon}, \bar{\varepsilon}, \check{\varepsilon}\}, L:=\max _{s \in[-M, M]} \phi^{\prime}(s)$.

Clearly, we have:

$$
\int_{B_{1-\varepsilon}} d \mu=\int_{0}^{1-\varepsilon} \int_{\partial B_{r}} \frac{2}{1-r^{2}} d r d \mu^{\prime}
$$

where $d \mu^{\prime}$ is the Riemannian area element on $\partial B_{r}(0<\varepsilon<1,0<r<1-\varepsilon)$. 
By $\left(H_{1}\right)^{*},(4.16)$ and $(4.20)$ we have

$$
\begin{aligned}
& \frac{1}{L} \int_{0}^{T} \int_{B_{1} \backslash B_{1-\tilde{\varepsilon}}} a(x)\left[\phi\left(v_{1}\right)-\phi\left(v_{2}\right)\right] \psi_{0}(x) d \mu d \tau \\
& \geq \frac{\bar{C}}{L} \int_{0}^{T} \int_{B_{1} \backslash B_{1-\tilde{\varepsilon}}} \underline{a}(1-|x|)\left[\phi\left(v_{1}\right)-\phi\left(v_{2}\right)\right]\left(1-|x|^{2}\right)^{n-1} d \mu d \tau \\
& =\frac{2 \bar{C}}{L} \int_{0}^{\tilde{\varepsilon}} \int_{\partial B_{1-\varepsilon}} \frac{\underline{a}(\varepsilon)}{\varepsilon(2-\varepsilon)}(2-\varepsilon)^{n-1} \varepsilon^{n-1} \int_{0}^{T}\left[\phi\left(v_{1}\right)-\phi\left(v_{2}\right)\right] d \mu^{\prime} d \varepsilon d \tau \\
& =\frac{2 \bar{C}}{L} \int_{0}^{\tilde{\varepsilon}} \frac{a(\varepsilon)}{\varepsilon}(2-\varepsilon)^{n-2} \varepsilon^{n-1} \varphi(\varepsilon) d \varepsilon \geq \frac{(2-\tilde{\varepsilon})^{n-2} \bar{C} \gamma}{L} \int_{0}^{\tilde{\varepsilon}} \frac{a}{\varepsilon}(\varepsilon) \\
& \varepsilon
\end{aligned}
$$

The previous inequalities and (4.19) yield

$$
\int_{0}^{T} \int_{B_{1}} a(x)\left[v_{1}(x, \tau)-v_{2}(x, \tau)\right] \psi_{0}(x) d \mu d \tau=\infty,
$$

in contrast with (4.18). Hence (4.13) follows. The proof is complete.

For any $\varepsilon \in\left(0, \varepsilon_{0} / 2\right)$ consider the auxiliary problem

$$
\begin{cases}a u_{t}=\Delta_{g}[\phi(u)] & \text { in } B_{1-\varepsilon} \times(0, T]=: H_{\varepsilon, T} \\ u=\zeta & \text { in } \partial B_{1-\varepsilon} \times(0, T) \\ u=u_{0} & \text { in } B_{1-\varepsilon} \times\{0\},\end{cases}
$$

where $\zeta \in L^{\infty}\left(\partial B_{1-\varepsilon} \times(0, T)\right)$.

Definition 4.3. A supersolution of problem (4.21) is a function $u \in C\left(B_{1-\varepsilon} \times\right.$ $(0, T])$ such that

$$
\begin{aligned}
\int_{0}^{\tau} \int_{\Omega_{1}}\left\{a u \partial_{t} \psi+\phi(u) \Delta_{g} \psi\right\} d \mu d t \leq & \int_{\Omega_{1}} a\left[u(x, \tau) \psi(x, \tau)-u_{0}(x) \psi(x, 0)\right] d \mu \\
& +\int_{0}^{\tau} \int_{\partial \Omega_{1} \cap \partial B_{1-\varepsilon}} \phi(\zeta)\left\langle\nabla_{g} \psi, \nu\right\rangle_{g} d \mu^{\prime} d t
\end{aligned}
$$

for any open set $\Omega_{1} \subseteq B_{1-\varepsilon}$ with smooth boundary $\partial \Omega_{1}, \tau \in(0, T], \psi \in$ $C^{2,1}\left(\overline{\Omega_{1}} \times[0, \tau]\right), \psi \geq 0, \psi=0$ in $\partial \Omega_{1} \times[0, \tau]$; here $\nu$ denotes the outer normal to $\Omega_{1}$ with $\|\nu\|_{g}=1$. Solutions and subsolutions are defined accordingly.

Since $\Delta_{g}$ is a uniformly elliptic operator in $B_{1-\varepsilon}$ for any $\varepsilon \in(0,1)$, existence, uniqueness and comparison results for problem (4.21) can be proved by standard methods.

Now we can prove Theorem 2.2.

Proof of Theorem 2.2. We give the proof replacing $\left(H_{1}\right)$ by the weaker condition $\left(H_{1}\right)^{*}$, introduced in Remark 2.4.

Let $u_{1}, u_{2}$ be any two bounded solutions of problem (1.1); set

$$
M:=\max \left\{\sup _{H_{T}}\left|u_{1}\right|, \sup _{H_{T}}\left|u_{2}\right|\right\} .
$$


For any $\varepsilon \in\left(0, \varepsilon_{0} / 2\right)$ let $u_{\varepsilon}$ be the unique solution of problem (4.21) with $\zeta \equiv-M$. By comparison results we have:

$$
-M \leq u_{\varepsilon} \leq u_{1} \quad \text { and }-M \leq u_{\varepsilon} \leq u_{2} \quad \text { in } \quad H_{\varepsilon, T} .
$$

By usual compactness arguments there exists a subsequence $\left\{u_{\varepsilon_{m}}\right\} \subseteq\left\{u_{\varepsilon}\right\}$ which converges uniformly in any compact subset of $\mathbb{H}^{n} \times(0, T]$. Set

$$
\underline{u}:=\lim _{m \rightarrow \infty} u_{\varepsilon_{m}} \text { in } \mathbb{H}^{n} \times(0, T] .
$$

The function $\underline{u}$ is a solution of problem (1.1); moreover, from (4.22) we obtain

$$
-M \leq \underline{u} \leq u_{1} \quad \text { and }-M \leq \underline{u} \leq u_{2} \quad \text { in } \quad H_{T} .
$$

Set $w=u_{1}$ or $w=u_{2}$ for simplicity. The conclusion will follow, if we show that

$$
\int_{0}^{T} \int_{\mathbb{H}^{n}}[\phi(w)-\phi(\underline{u})] F d \mu d t=0
$$

for any $F \in C_{0}^{\infty}\left(\mathbb{H}^{n}\right)$.

In fact, in view of assumption $\left(H_{0}\right)-(i i)$ and the arbitrariness of $F$, (4.24) implies

$$
u_{1}=\underline{u}=u_{2} \quad \text { in } \quad H_{T}
$$

whence the conclusion.

Let us prove equality (4.24). Without loss of generality, we suppose supp $F \subseteq\left(B_{1-\varepsilon_{0}}\right), F \geq 0, F \not \equiv 0$.

In view of the inequality $w \geq \underline{u}$ (see (4.23)), arguing as in the proof of Lemma 4.2, we obtain

$$
\begin{gathered}
\int_{B_{1-\varepsilon}} a(x)[w(x, \tau)-\underline{u}(x, \tau)] \psi_{\varepsilon}(x) d \mu+\int_{0}^{\tau} \int_{B_{1-\varepsilon}}[\phi(w)-\phi(\underline{u})] F(x) d \mu d t \\
=-\int_{0}^{\tau} \int_{\partial B_{1-\varepsilon}}\{\phi(w)-\phi(\underline{u})\}\left\langle\nabla_{g} \psi_{\varepsilon}, \nu_{\varepsilon}\right\rangle_{g} d \mu^{\prime} d t
\end{gathered}
$$

where $\psi_{\varepsilon}$ denotes the solution of (4.1), $\varepsilon \in\left(0, \varepsilon_{0} / 2\right), \tau \in(0, T]$. Since $F \geq 0$, $\psi_{\varepsilon} \geq 0, w \geq \underline{u}$ and $\left(H_{0}\right)-(i i)$ holds true, equality (4.26) with $\tau=T$ gives

$$
\begin{aligned}
& \int_{0}^{T} \int_{B_{1}}[\phi(w)-\phi(\underline{u})] F(x) d \mu d t \\
& \quad \leq \liminf _{\varepsilon \rightarrow 0}\left|\int_{0}^{T} \int_{\partial B_{1-\varepsilon}}\{\phi(w)-\phi(\underline{u})\}\left\langle\nabla_{g} \psi_{\varepsilon}, \nu_{\varepsilon}\right\rangle_{g} d \mu^{\prime} d t\right| .
\end{aligned}
$$

Hence, if we can prove that

$$
\liminf _{\varepsilon \rightarrow 0}\left|\int_{0}^{T} \int_{\partial B_{1-\varepsilon}}\{\phi(w)-\phi(\underline{u})\}\left\langle\nabla_{g} \psi_{\varepsilon}, \nu_{\varepsilon}\right\rangle_{g} d \mu^{\prime} d t\right|=0,
$$

the conclusion follows.

Equality (4.28) is just a consequence of (4.13) with $v_{1}=w$ and $v_{2}=u$ together with (4.3). The proof is complete. 


\section{Proof of Theorem 2.5}

We shall consider equation

$$
\Delta_{g} V=-a \text { in } \mathbb{H}^{n} .
$$

Let us give the following

Definition 5.1. A supersolution to equation (5.1) is a function $U \in C\left(B_{1}\right)$ such that

$$
\int_{\Omega_{1}} U \Delta_{g} \psi d \mu \leq \int_{\partial \Omega_{1}} U\left\langle\nabla_{g} \psi, \nu\right\rangle_{g} d \mu^{\prime}-\int_{\Omega_{1}} a \psi d \mu
$$

for any open set $\Omega_{1} \subseteq B_{1}$ with smooth boundary $\partial \Omega_{1}, \bar{\Omega}_{1} \subseteq B_{1}, \psi \in$ $C^{2}\left(\overline{\Omega_{1}}\right), \psi \geq 0, \psi=0$ in $\partial \Omega_{1}$; here $\nu$ denotes the outer normal to $\Omega_{1},\|\nu\|_{g}=1$. Subsolutions and solutions of equation (5.1) are defined accordingly.

The following lemma will be used.

Lemma 5.2. Let assumption $\left(\mathrm{H}_{2}\right)$ be satisfied. Then there exists a positive supersolution $V$ to equation (5.1) such that

$$
\lim _{|x| \rightarrow 1} V(x)=0 .
$$

Proof of Lemma 5.2. Let

$$
\beta \in\left(0, \min \left\{\frac{n-1}{2},-\alpha\right\}\right], \quad C:=\frac{2 C_{2}}{\beta} .
$$

Define

$$
V(x):=C\left(1-|x|^{2}\right)^{\beta} \quad\left(x \in \mathbb{H}^{n}\right) .
$$

For any $x \in \mathbb{H}^{n}$ we have:

$$
\begin{gathered}
\frac{\partial V(x)}{\partial x_{i}}=-2 C \beta\left(1-|x|^{2}\right)^{\beta-1} x_{i}, \\
\frac{\partial^{2} V(x)}{\partial x_{i} \partial x_{j}}=2 C \beta\left(1-|x|^{2}\right)^{\beta-2}\left[2(\beta-1) x_{i} x_{j}-\left(1-|x|^{2}\right) \delta_{i j}\right],
\end{gathered}
$$

where $i, j=1, \ldots, n$. From (5.4) and the fact that $n \geq 2$ we obtain:

$$
\begin{aligned}
\Delta_{g} V(x) & =\frac{C}{2} \beta\left(1-|x|^{2}\right)^{\beta}\left[2(\beta-1)|x|^{2}-n\left(1-|x|^{2}\right)-2(n-2)|x|^{2}\right] \\
& \leq \frac{C}{2} \beta\left(1-|x|^{2}\right)^{\beta}\left[(2 \beta+2-n)|x|^{2}-n\right] \\
& \leq \frac{C}{2} \beta\left(1-|x|^{2}\right)^{\beta}\left[(2 \beta+1-n)|x|^{2}-n+1\right] \\
& \leq-C_{2}\left(1-|x|^{2}\right)^{\beta} .
\end{aligned}
$$

This inequality, combined with hypothesis $\left(H_{2}\right)$ and (5.4), implies that

$$
\Delta_{g} V(x) \leq-a(x) \text { for any } x \in \mathbb{H}^{n} .
$$

Moreover, clearly, condition (5.3) is satisfied. Hence the proof is complete. 
Observe that for any $A \in \operatorname{Lip}([0, T])$ the derivative $A^{\prime}$ exists almost everywhere in $[0, T]$ and belongs to $L^{\infty}((0, T))$.

For any $\varepsilon \in\left(0, \varepsilon_{0} / 2\right)$ we will make use of the following auxiliary problems

$$
\begin{cases}a \partial_{t} u=\Delta_{g}[\phi(u)] & \text { in } H_{\varepsilon, T} \\ u=\phi^{-1}\left(A^{\prime}\right) & \text { in } \partial B_{1-\varepsilon} \times(0, T) \\ u=u_{0} & \text { in } B_{1-\varepsilon} \times\{0\}\end{cases}
$$

and

$$
\begin{cases}\Delta_{g} U=f & \text { in } B_{1-\varepsilon} \\ U=\gamma & \text { in } \partial B_{1-\varepsilon}\end{cases}
$$

where $f \in C\left(B_{1-\varepsilon}\right)$ and $\gamma \in C\left(\partial B_{1-\varepsilon}\right)$.

Definition 5.3. A supersolution of problem (5.6) is a function $U \in C\left(B_{1-\varepsilon}\right)$ such that

$$
\int_{\Omega_{1}} U \Delta_{g} \psi d \mu \leq \int_{\Omega_{1}} f \psi d \mu+\int_{\partial \Omega_{1} \cap \partial B_{1-\varepsilon}} \gamma\left\langle\nabla_{g} \psi, \nu\right\rangle_{g} d \mu^{\prime}
$$

for any open set $\Omega_{1} \subseteq B_{1-\varepsilon}$ with smooth boundary $\partial \Omega_{1}, \psi \in C^{2}\left(\overline{\Omega_{1}}\right), \psi \geq$ $0, \psi=0$ in $\partial \Omega_{1}$; here $\nu$ denotes the outer normal to $\Omega_{1}$ with $\|\nu\|_{g}=1$. Solutions and subsolutions are defined accordingly.

Proof of Theorem 2.5. For any $\varepsilon \in\left(0, \varepsilon_{0} / 2\right)$ let $u_{\varepsilon}$ be the unique solution to problem (5.5); then by comparison results we have

$$
\left|u_{\varepsilon}\right| \leq \sup _{\mathbb{H}^{n}}\left|u_{0}\right|+\max _{-\left\|A^{\prime}\right\|_{\infty} \leq r \leq\left\|A^{\prime}\right\|_{\infty}}\left|\phi^{-1}(r)\right|=: M \quad \text { in } H_{\varepsilon, T} .
$$

By usual compactness arguments there exists a subsequence $\left\{u_{\varepsilon_{m}}\right\} \subseteq\left\{u_{\varepsilon}\right\}$, which converges uniformly in any compact subset of $\mathbb{H}^{n} \times(0, T]$ to a solution $u$ of problem (1.1).

Define $U$ as in (2.6) and

$$
U_{\varepsilon}(x, t):=\int_{0}^{t} \phi\left(u_{\varepsilon}(x, \tau)\right) d \tau \quad\left((x, t) \in H_{\varepsilon, T}\right) .
$$

Observe that $U_{\varepsilon_{m}} \rightarrow U$ in $\mathbb{H}^{n} \times(0, T]$ as $m \rightarrow \infty$.

It is straightforward to show that for any $t \in(0, T]$ the function $U_{\varepsilon}(\cdot, t)$ satisfies the problem (5.6) with $f=-\rho\left[u_{0}-u(\cdot, t)\right], \gamma=A(t)$. In fact, by Definition 4.3 we obtain

$$
\begin{aligned}
\int_{\Omega_{1}} U_{\varepsilon}(x, t) \Delta_{g} \psi(x) d x= & \int_{\Omega_{1}} a(x)\left[u_{\varepsilon}(x, t)-u_{0}(x)\right] \psi(x) d \mu \\
& +\int_{\partial \Omega_{1} \cap \partial B_{1-\varepsilon}} A(t)\left\langle\nabla_{g} \psi(x), \nu(x)\right\rangle_{g} d \mu^{\prime}
\end{aligned}
$$

for any $\Omega_{1}$ and $\psi=\psi(x)$ as in Definition 5.3 and $t \in(0, T]$. 
We shall prove that

$$
-2 M V(x)+A(t) \leq U(x, t) \leq 2 M V(x)+A(t) \quad\left((x, t) \in H_{T}\right),
$$

where $V$ is the supersolution introduced in Lemma 5.2.

From (5.3) and (5.10) the equality (2.5), thus the conclusion follows.

It remains to prove (5.10). It is easily seen that for any fixed $t \in(0, T]$ the function $2 M V-U_{\varepsilon}+A$ is a supersolution, while the function $-2 M V-U_{\varepsilon}+A$ is a subsolution of problem (5.6) with $f=\gamma \equiv 0$ for any $\varepsilon \in\left(0, \varepsilon_{0} / 2\right)$. In fact, fix any $\Omega_{1}, \psi$ as in Definition 5.3 and $t \in(0, T]$. Then by (5.9) and (5.2) we have

$$
\begin{aligned}
\int_{\Omega_{1}}(2 M V & \left.-U_{\varepsilon}+A\right) \Delta_{g} \psi d x \leq \int_{\partial \Omega_{1} \backslash \partial B_{1-\varepsilon}}\left(2 M V-U_{\varepsilon}+A\right)\left\langle\nabla_{g} \psi, \nu\right\rangle_{g} d \mu^{\prime} \\
& +\int_{\partial \Omega_{1} \cap \partial B_{1-\varepsilon}} 2 M V\left\langle\nabla_{g} \psi, \nu\right\rangle_{g} d \mu^{\prime}-\int_{\Omega_{1}}\left(2 M+u_{\varepsilon}-u_{0}\right) a \psi d x=
\end{aligned}
$$

for any $\varepsilon \in\left(0, \varepsilon_{0}\right)$.

Notice that

$$
2 M V \geq 0 \quad \text { on } \quad \partial B_{1-\varepsilon}, 2 M \geq u_{0}-u_{\varepsilon} \quad \text { in } \quad \Omega_{1}
$$

for any $\varepsilon \in\left(0, \varepsilon_{0}\right)$. Moreover, it is easily checked that

$$
\left\langle\nabla_{g} \psi, \nu\right\rangle_{g} \leq 0 \quad \text { on } \quad \partial \Omega_{1} .
$$

From (5.11) to (5.13) we obtain

$$
\int_{\Omega_{1}}\left(2 M V-U_{\varepsilon}+A\right) \Delta_{g} \psi d \mu \leq \int_{\partial \Omega_{1} \backslash \partial B_{1-\varepsilon}}\left(2 M V-U_{\varepsilon}+A\right)\left\langle\nabla_{g} \psi, \nu\right\rangle_{g} d \mu^{\prime}
$$

for any $\varepsilon \in\left(0, \varepsilon_{0}\right)$. This shows that the function $2 M V-U_{\varepsilon}+A$ is a supersolution to problem (5.6) with $f=\gamma \equiv 0$ for any $\varepsilon \in\left(0, \varepsilon_{0}\right)$ (see Definition 5.3). It is similarly seen that $-2 M V-U_{\varepsilon}+A$ is a subsolution of the same problem for any $\varepsilon \in\left(0, \varepsilon_{0}\right)$.

By comparison principles we obtain

$$
-2 M V(x)+A(t) \leq U_{\varepsilon}(x, t) \leq 2 M V(x)+A(t) \quad\left((x, t) \in\left[H_{\varepsilon, T}\right)\right.
$$

for any $\varepsilon \in\left(0, \varepsilon_{0}\right)$. This implies (5.10), thus the proof is complete.

To prove Theorem 2.8 we need a preliminary result.

Lemma 5.4. Let there exist a nonnegative supersolution to equation (5.1) such that

$$
\inf _{\mathbb{H}^{n}} V=0 .
$$

Then there exists a sequence $\left\{x_{m}\right\} \subseteq \mathbb{H}^{n},\left|x_{m}\right| \rightarrow 1$ as $m \rightarrow \infty$ such that

$$
\lim _{m \rightarrow \infty} V\left(x_{m}\right)=0 .
$$

Lemma 5.4 can be proved arguing as in the proof Lemma 2.6 in [18]. Proof of Theorem 2.8. The function

$$
V(x):=\int_{\mathbb{H}^{n}} G_{g}(x, y) a(y) \quad d \mu(y)-\inf _{x \in \mathbb{H}^{n}}\left(\int_{\mathbb{H}^{n}} G_{g}(x, y) a(y) d \mu(y)\right) \quad\left(x \in \mathbb{H}^{n}\right)
$$


is a nonnegative solution to equation (5.1) satisfying condition (5.15) (see [8]). Then by Lemma 5.4 there exists a sequence $\left\{x_{m}\right\} \subseteq \mathbb{H}^{n},\left|x_{m}\right| \rightarrow 1$ as $m \rightarrow \infty$ such that $V\left(x_{m}\right) \rightarrow 0$ as $m \rightarrow \infty$. The conclusion follows repeating the proof of Theorem 2.5.

\section{References}

[1] Benedetti, R., Petronio, C.: Lectures on hyperbolic geometry. Springer, Berlin (1992).

[2] Benilan, P.H., Crandall, M.G., Pierre, M.: Solutions of the porous medium equation in $\mathbb{R}^{n}$ under optimal conditions on initial values. Indiana Univ. Math. J. 33, 51-87 (1984)

[3] Birindelli, I., Mazzeo, R.: Symmetry for solutions of two phase semilinear elliptic equations on hyperbolic space. Indiana Univ. Math. J. 58, 2347-2368 (2009)

[4] Brezis, H., Crandall, M.G.: Uniqueness of solutions of the initial-value problem for $u_{t}-\Delta \varphi(u)=0$. J. Math. Pures Appl. 58, 153-163 (1979)

[5] Davies, E.B.: Heat Kernel and Spectral Theory. Cambridge University Press, Cambridge (1989)

[6] Eidus, D., Kamin, S.: The filtration equation in a class of functions decreasing at infinity. Proc. Am. Math. Soc. 120, 825-830 (1994)

[7] Grigoryan, A.: On the existence of a Green function on a manifold. Russ. Math. Surv. 38, 190-191 (1983)

[8] Grigoryan, A.: Analytic and geometric background of recurrence and nonexplosion of the Brownian motion on Riemannian manifolds. Bull. Am. Math. Soc. 36, 135-249 (1999)

[9] Grigoryan, A.: Heat kernels on weighted manifolds and applications. Cont. Math. 398, 93-191 (2006)

[10] Grigoryan, A., Noguchi, M.: The heat kernel on hyperbolic space. Bull. Lond. Math. Soc. 30, 643-650 (1998)

[11] Guedda, M., Hilhorst, D., Peletier, M.A.: Disappearing interfaces in nonlinear diffusion. Adv. Math. Sci. Appl 7, 695-710 (1997)

[12] Kalashnikov, A.S.: The Cauchy problem in a class of growing functions, Vestnik Moscow 6, pp. 17-27 (1963) (in Russian).

[13] Kamin, S., Kersner, R., Tesei, A.: On the Cauchy problem for a class of parabolic equations with variable density. Rend. Mat. Acc. Lincei 9, 279-298 (1998)

[14] Kamin, S., Pozio, M.A., Tesei, A.: Admissible conditions for parabolic equations degenerating at infinity. St. Petersburg Math. J. 19, 239-251 (2008)

[15] Kamin, S., Rosenau, P.: Non-linear diffusion in a finite mass medium. Commun. Pure Appl. Math. 35, 113-127 (1982) 
[16] Kumaresan, S., Prajapat, J.: Serrin's result for hyperbolic space and sphere. Duke Math. J., 17-29 (1998)

[17] Pozio, MA., Punzo, F., Tesei, A.: Uniqueness and nonuniqueness of solutions to parabolic problems with singular coefficients. Discret. Contin. Dyn. Syst. A. 30, 891-916 (2011)

[18] Pozio, M.A., Tesei, A.: On the uniqueness of bounded soutions to singular parabolic problems. Discret. Contin. Dyn. Syst. 13, 117-137 (2005)

[19] Punzo, F.: On the Cauchy problem for nonlinear parabolic equations with variable density. J. Evol. Equ. 9, 429-447 (2009)

[20] Punzo, F.: Support properties of solutions to nonlinear parabolic equations with variable density in the hyperbolic space. Discret. Contin. Dyn. Syst. S. doi:10.3934/dcdss.2012.5.xx

[21] Reyes, G., Vazquez, J.L.: The Cauchy problem for the inhomogeneous porous medium equation. Netw. Heterog. Media 2, 337-351 (2006)

F. Punzo

Dipartimento di Matematica "G. Castelnuovo"

Università di Roma "La Sapienza"

P.le A. Moro 5

00185 Rome

Italy

e-mail: punzo@mat.uniroma1.it

Received: 20 January 2011.

Accepted: 20 September 2011. 